\title{
An unusual cause of
}

\section{gastrointestinal bleeding}

\section{in a young girl}

A I2-year-old girl was referred to our gastroenterology unit for further examination after 2 self-limited episodes of upper gastrointestinal (GI) bleeding with hematemesis and melena. She had taken no medications at all during the month before her hospital admission.

Laboratory and endoscopic investigation revealed no upper GI pathology, but close clinical observation found soft, yellowish papules joined into a plaque $8 \mathrm{~cm}$ in length on her lumbar aspect (Fig. I). We hypothesized a diagnosis of pseudoxanthoma elasticum (PXE) and took a skin biopsy.

Histological examination showed alterations of the skin's elastic fibres, which confirmed our provisional diagnosis. Further investigations to evaluate the involvement of other systems detected angioid streaks on both sides of her ocular fundus. Coronary angiography yielded no abnormal findings.

One year later, no additional signs of systemic involvement were found, and she had experienced no further GI bleeding.

PXE is an inherited, multisystem disorder that affects primarily the skin, eyes and vascular system. ${ }^{1}$ Its estimated prevalence is I in $70000-\mathrm{IO0} 000$, with a 2:I female preponderance. The defect has been mapped to the ABCC6 gene on chromosome r6pr3.I; the gene encodes for the cellular transport protein ABCC6/MRP6, giving rise to the concept of PXE as a systemic metabolic disorder rather than a purely structural disorder of the connective tissue. ${ }^{2}$

Skin involvement is the most common manifestation: typically yellowish papules, mainly at the neck, axillae, abdomen and thighs, with a tendency to join into plaques. The histopathological features of the skin lesions are mineralization and fragmentation of the elastic fibres. Similar fibre changes occur in the blood vessels, Bruch's membrane of the eye, and other organs.



Fig. 1: Yellowish soft papules, joined into a plaque with a maximal length of $8 \mathrm{~cm}$, on the patient's lower back.

GI bleeding, usually recurrent and of gastric origin, reportedly occurs in I $3 \%$ of affected patients. The vessel ruptures are thought to occur from the degeneration of elastic fibres in arterial walls. When viewed endoscopically, the stomach may look normal, have a distinctive yellow "cobblestone" appearance, or exhibit raised nodular submucosal lesions.

Patients with PXE often have a normal life span, depending on the extent of systemic involvement. The most potentially serious complication is accelerated arteriosclerosis - arterioslerotic heart disease and hypertension have been reported in patients as young as 4 years of age $^{1}$ - but it is visual complications that most affect quality of life. ${ }^{2}$

Early diagnosis is paramount: because many of the pathological changes are irreversible, prophylactic measures must be taken to minimize the risk of complications. ${ }^{3}$ In particular, laser photocoagulation should be used to prevent retinal hemorrhage; the patient's risk factors for cardiovascular disease should be treated; and platelet inhibitors and warfarin must be avoided. ${ }^{3}$ Although the parents of our patient refused the option, plastic surgery can remove skin lesions that become a cosmetic problem.
Patients with GI bleeding of unknown origin must be carefully examined physically and observed, not only for the typical yellowish papules of PXE, but for signs of any of several other skin diseases that may have GI involvement and cause hematemesis. The most common of these include melanoma that has metastasized into the GI tract; blue rubber-bleb nevus syndrome, which often affects the internal organs as well as the skin and mucosae; Kaposi's sarcoma; and visceral neurofibromatosis (Recklinghausen's disease).

\section{Emiliano Antiga \\ Lucilla Melani \\ Marzia Caproni \\ Paolo Fabbri \\ Department of Dermatological \\ Sciences \\ University of Florence \\ Florence, Italy}

Competing interests: None declared.

\section{REFERENCES}

I. Hu X, Plomp AS, van Soest S, et al. Pseudoxanthoma elasticum: a clinical, histopathological, and molecular update. Surv Ophtalmol 2003;48:424-38.

2. Bercovitch L, Terry P. Pseudoxanthoma elasticum 2004. J Am Acad Dermatol 2004;5I(I Suppl):Si3-4.

3. Laube S, Moss C. Pseudoxanthoma elasticum. Arch Dis Child 2005;90:754-6. 